\title{
Analysis and forecasting of crude oil price based on the variable selection-LSTM integrated model
}

\author{
Quanying Lu', Shaolong Sun ${ }^{2}$, Hongbo Duan ${ }^{3^{*}}$ and Shouyang Wang ${ }^{1,3}$ \\ From 1st Energy Informatics.Academy Conference Asia \\ Beijing, China . 29-30 May 2021
}

\author{
* Correspondence: hbduan@ucas.ac. \\ $\mathrm{cn}$ \\ ${ }^{3}$ School of Economics and \\ Management, University of Chinese \\ Academy of Sciences, Beijing \\ 100190, China \\ Full list of author information is \\ available at the end of the article
}

\begin{abstract}
In recent years, the crude oil market has entered a new period of development and the core influence factors of crude oil have also been a change. Thus, we develop a new research framework for core influence factors selection and forecasting. Firstly, this paper assesses and selects core influence factors with the elastic-net regularized generalized linear Model (GLMNET), spike-slab lasso method, and Bayesian model average (BMA). Secondly, the new machine learning method long short-term Memory Network (LSTM) is developed for crude oil price forecasting. Then six different forecasting techniques, random walk (RW), autoregressive integrated moving average models (ARMA), elman neural Networks (ENN), ELM Neural Networks (EL), walvet neural networks (WNN) and generalized regression neural network Models (GRNN) were used to forecast the price. Finally, we compare and analyze the different results with root mean squared error (RMSE), mean absolute percentage error (MAPE), directional symmetry (DS). Our empirical results show that the variable selection-LSTM method outperforms the benchmark methods in both level and directional forecasting accuracy.
\end{abstract}

Keywords: Crude oil price, GLMNET, BMA, Spike-slab lasso, LSTM

\section{Springer Open}

\section{Introduction}

Since 2014, the international crude oil price has experienced the most significant volatility since the 2018 financial crisis. The oil market has taken on new features that affect the development of the global economy, national strategic security, and investor sentiment significantly. Especially as the primary alternative energy resources, the US tight oil production has been significant macroeconomic effects on the oil price (Kilian, 2017). In 2014, US shale oil producers plundered market share, leading to a change in global crude oil supply and demand balance. According to EIA, US shale oil production increased from 4.96 million barrels per day in 2017 to 5.59 million barrels per day in 2022. In addition, there are geopolitical events, trade frictions, and OPEC's agreement have occurred in recent years,

(c) The Author(s). 2021 Open Access This article is licensed under a Creative Commons Attribution 4.0 International License, which permits use, sharing, adaptation, distribution and reproduction in any medium or format, as long as you give appropriate credit to the original author(s) and the source, provide a link to the Creative Commons licence, and indicate if changes were made. The images or other third party material in this article are included in the article's Creative Commons licence, unless indicated otherwise in a credit line to the material. If material is not included in the article's Creative Commons licence and your intended use is not permitted by statutory regulation or exceeds the permitted use, you will need to obtain permission directly from the copyright holder. To view a copy of this licence, visit http://creativecommons.org/licenses/by/4.0/. 
causing the volatility of oil price. The internal and external environment of the oil market is changing, and the influencing factors have become diverse and complex. As the factors affecting international oil prices become more and more complex, it becomes difficult to capture practical factors and predict oil prices. Many past kinds of literature about crude oil price forecasting show that the forecasting results are sensitive to the modeling sample data frequency and data interval selection (Yu et al., 2019; Yu et al., 2008a; Zhang et al., 2015). As a strategic resource, crude oil plays a vital role in national energy security.

Meanwhile, with the financial properties of crude oil strengthened gradually, the volatility of crude oil prices is bound to affect oil companies' earnings and investor behavior. Therefore, systematic analysis of the characteristics of complex international oil markets and accurate capture of the new trend in international oil prices are critical. However, as the linkage between the markets, the uncertainty of the world economy and energy, the influence factors of oil price have become complex. It is difficult to point out which factors have the dominant effect on the oil price. If all possible oil price factors are added into the existing forecast model, it may lead to over-fitting problems, which will affect the forecast results. How to forecast crude oil prices in a new and effective method is one problem that academics and practitioners are very concerned about all the time. It can provide reference and theoretical support for the formulation of national energy security strategy and enterprise avoidance of market risks. To better analyze the changing trend of the crude oil market, it is necessary to determine the main factors affecting the price, determine the impact of each factor on price, and establish a forecasting model finally.

The research on the prediction of international oil price has always been a hot topic. A large number of papers with theoretical and practical application value have appeared. We make a simple review from two aspects of influencing factors and forecasting methods as follow:

\section{Influencing factor}

Most of the research has divided the influence factors of crude oil price into supply and demand, finance factor, technology (Hamilton, 2009a; Kilian \& Murphy, 2014; Zhang et al., 2017; Wang et al., 2015; Tang et al., 2012).

\section{Supply and demand}

As the fundamental factor, supply and demand have been the main factors affecting oil prices. Supply and demand changes have always been the fundamental factors affecting the long-term trend of oil prices. (Hamilton, 2009b) analyzed the drivers of oil prices and argued that the main reason for the rise in oil prices in 2007-2008 was the global demand for production. (Kilian, 2009) developed a structural VAR model to explain the global crude oil price fluctuation and understand the reaction of the US economy associated with oil price volatility. The crude oil price was decomposed into three components: crude oil supply shock, the shocks to the global demand for all industrial commodities, and the demand shock to the global crude oil market. However, in recent years, with the development of alternative energy sources, the worldwide supply and demand structure of crude oil has changed. (Kilian, 2017) reported the increased U.S. tight oil production not only reduced demand for oil in the rest of the world and 
lowering the Brent oil price but also caused other countries to cut back on their oil imports, lowering global oil demand.

\section{Global economic development}

Global economic development is a manifestation of supply and demand (Doroodian \& Boyd, 2003; Sadorsky, 1999; Barsky \& Kilian, 2001). (Kilian \& Hicks, 2013) measured the global demand shock directly by correcting the real gross domestic product (GDP) growth forecast. The results showed that the forecast was associated with unexpected growth in emerging economies during the 2003 to 2008 period. These surprises were associated with a hump-shaped response of the real price of oil that reaches its peak after 12-16 months. The global real economic activity has always been considered to impact the changes in oil price significantly. (Özbek \& Özlale, 2010) researched the relationship between global economic and oil prices with trend and cycle decomposition. They found that economic shock has a lasting effect on oil prices, which were considered mainly to be supply-side driven.

\section{Financial factor}

In addition to commodity attributes, crude oil also has financial attributes. The longterm trend of crude oil price is determined by the commodity attributes, which are affected by the supply and demand factors generated by the real economy; the shortterm fluctuations of crude oil price are determined by the financial attributes, which are influenced by market expectations and speculative transactions. The financial factor mainly includes speculation factor, exchange rate and some other financial index, which connect the stock market and monetary market with the crude oil price (Narayan et al., 2010; Zhang, 2013; Reboredo, 2012; Cifarelli \& Paladino, 2010). (Kilian \& Murphy, 2014) developed a structural model to estimate the speculative component of oil price through the inventory data and found it played an important role during earlier oil price shock periods, including 1979, 1986 and 1990. (Sari et al., 2010) estimated the comovement and information transmission among oil price, exchange rate and the spot prices of four precious metals (gold, silver, platinum, and palladium). Investors could diversify their investment risk by investing in precious metals, oil, and euros.

\section{Technology factor}

The Crack spread is defined as the price difference between crude oil and its refined oil, reflecting the supply and demand relationship between the crude oil market and its refined product market (Wang et al., 2015). (Murat \& Tokat, 2009) used the random walk model (RWM) as a benchmark to compare the crack spread futures and crude oil futures and found the crack future could forecast the movements of oil spot price as reasonable as the crude oil futures. (Baumeister et al., 2013) selected crack spread as one of the variables to forecast crude oil prices, and the studies suggested it was an influential prediction factor.

\section{Forecast method}

Except for the influence factors, researchers are also very concerned about the forecast methods for improving forecast accuracy. The four main forecast method categories: time series models, econometric models, qualitative methods and artificial intelligence 
techniques are used in oil price modeling and forecasting (Wang et al., 2016; Charles \& Darné, 2017; Yu et al., 2015; Sun et al., 2019; Suganthi \& Samuel, 2012; Zhang et al., 2008; Valgaev et al., 2020). The autoregressive integrated moving average (ARIMA) and exponential smoothing (ETS) are the most widely used time series forecasting model, and they are usually used as the benchmark models (Wang et al., 2018; Chai et al., 2018; Zhu et al., 2017). In addition, the econometric models and qualitative methods like the generalized autoregressive conditional heteroskedastic model (GARCH), the vector autoregression model (VAR), the state-space models and the threshold models are also widely used (Kilian, 2010; Wang \& Sun, 2017; Zhang \& Wei, 2011; Ji \& Fan, 2016; Drachal, 2016).

However, with the increasing of the data volume and influence factors complex, traditional models failed in predicting accurately. The machine learning forecasting method presents its superiority and mostly outperform traditional approaches when tested with empirical results, especially in dealing with the nonlinear problem and short-term prediction. Such as support vector machines (SVM), artificial neural networks (ANNs), genetic algorithms (GA) and wavelet analysis are introduced into oil price forecasting in recent years. For example, (Zhao et al., 2017) proposed the stacked denoising autoencoders model (SDAE) for oil price forecasting. Empirical results of the proposed method proved the effectiveness and robustness statistically. (Xiong et al., 2013) developed an integrated model EMD-FNN-SBN, which is the empirical mode decomposition (EMD) based on the feed-forward neural network (FNN) modeling framework incorporating the slope-based method (SBM). The results indicate this model using the (multiple-input multiple-output) MIMO strategy is the best in prediction accuracy.

During the last decades, more and more factors and models have been introduced, estimated and validated. Several different factors can address the oil price forecasting problem from the empirical and theoretical vision. Many researchers always select general factors and models directly, regardless of which indicators are the actual core variables. Especially with the expansion of data and quantitative indicators, variable selection becomes more and more critical. In recent years, there are some papers begin to extract core factors before forecasting. Even though there are some variable selection processes in some machine learning methods, they are all nested in the forecasting and just for the robustness of the model (Drezga \& Rahman, 1998; May et al., 2008; Korobilis, 2013; Huang et al., 2014). There are fewer papers devoted to variable selection before predicting. For example, (Chai et al., 2018) used the Bayesian model average method for influence variable selection before establishing the oil price forecasting model. (Zhang et al., 2019) accurately screen out the main factors affecting oil price by using an elastic network model and LASSO shrinkage in the case of many predictive variables but relatively few data. The main factors influencing oil price forecast are studied from the Angle of variable selection. Secondly, the accuracy and robustness of the elastic network model and the LASSO contraction method in predicting oil prices are comprehensively verified using a variety of robustness tests. The results show that the LASSO contraction and elastic network model outperforms other standard oil price forecasting models. An investor who allocates assets based on the predictions of these two methods can achieve a more substantial return than other oil price forecasting models.

In this paper, we develop an integrated model with a new machine learning method for crude oil price forecasting based on core factor selection. This paper contributes to 
the variable selection and machine learning method in oil price forecasting. In the process of variable selection, we introduce three approaches with different advantages for comparison analysis and forecasting, elastic-net regularized generalized linear model, Bayesian model average and spike-slab lasso method. In addition, we combine them with a new machine learning method long short-term memory (LSTM) model for oil price forecasting. Finally, random walk (RW), autoregressive integrated moving average models (ARMA), elman neural Networks (ENN), ELM Neural Networks (EL), Walvet Neural Networks (WNN) and generalized regression neural network (GRNN) Models were used to forecast the price. Finally, we compare and analyze the different results with root mean squared error (RMSE), mean absolute percentage error (MAPE), directional symmetry (DS). The research framework is shown in Fig.1.

The structure of the paper is as follows: Section 1 reviews the related literature including influencing factors and forecast methods. Section 2 introduces the data. Section 3 develops the technique. Section 4 presents the empirical analysis. Finally, Section 5 concludes the paper and outlines future work.

\section{Dataset}

According to the above literature, we selected 30 variables from supply and demand, inventory, financial market, technical aspects as the initially chosen variables (Hamilton, 2009a; Kilian \& Murphy, 2014; Zhang et al., 2017; Wang et al., 2015; Tang et al., 2012), and we choose the real monthly West Texas Intermediate (WTI) crude oil price as the dependent variable. The interval of the data is from January 2000 to December 2017. For data stability, we used the return rate of data. The crude oil production, consumption structure and replacement cost as the related variables measure the supply index. The demand index includes crude oil consumption and global economic development, a measure of real global economic activity.

Meanwhile, we select the total OECD petroleum inventory, U.S. crude Oil inventory (total, SPR, and Non-SPR) as the inventory index. In addition, the related variables are selected from speculation, monetary, stock, commodity market as the financial factor

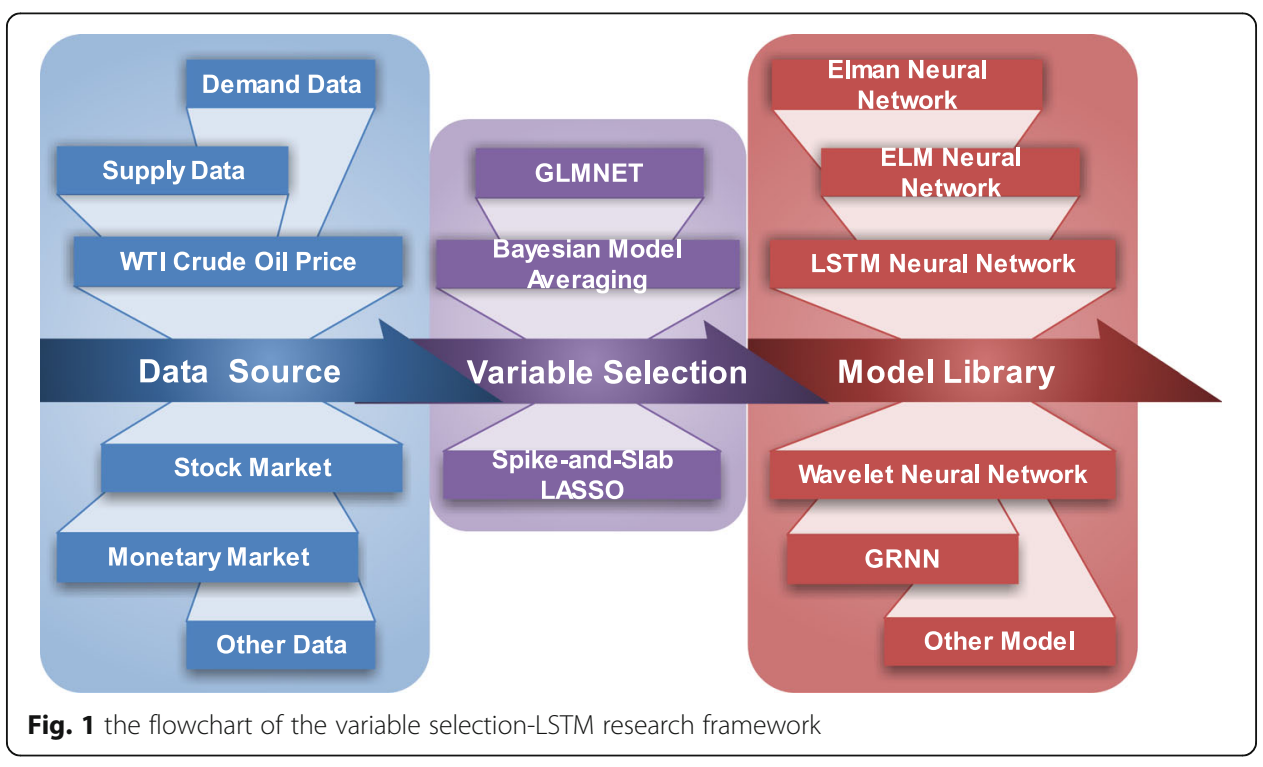


index. Finally, we calculated the WTI-Brent spot price spread, actual value of the WTI crack spread and Brent crack spread: actual value as the technical indicators. In Table 1, we describe each variable and its corresponding data sources.

\section{Theoretical background}

As the crude oil market is very complex and has various uncertain determinants, we must select the core influence factors first before establishing forecasting models. The main four variable selection methods are significance test (forward and backward stepwise regression), information criteria (AIC BIC), principal component factor analysis model, lasso regression, ridge regression, and other punitive models (Castle et al., 2009). It is hard to tell which the best is because each has its own strong and weak points. Thus, we introduce three different methods to select core influence factors of crude oil price, which are elastic-net regularized generalized linear Models (GIMNET), spike-slab lasso method (SSL) and Bayesian model averaging (BMA). These three methods are effective variable selection methods and they are all improvements on the existing mature models (LASSO, Ridge regression). Thus, we use these new methods for variable selection (Zou \& Hastie, 2005; Friedman et al., 2010).

\section{Variable selection}

\section{The elastic-net regularized generalized linear Models (GLMNET)}

Zou and Hastie (2005) (Zou \& Hastie, 2005) proposed the elastic-net method for variable selection, which is considered to be the best contraction method for handling multicollinearity and variable screening, and its loss precision is not too great. Their simulation results showed that the elastic net outperformed the Lasso in terms of prediction accuracy. Like the Lasso, the elastic net simultaneously does automatic variable selection and continuous shrinkage, and it can select groups of correlated variables. On the one hand, it achieves the purpose of ridge regression to select essential features; on the other hand, it removes features that have little influence on dependent variables, like Lasso regression, and achieves good results, especially when the sample size $\mathrm{n}$ is smaller than the number of predictors. (The specific formula refers to (Zou \& Hastie, 2005)) In this paper, we choose the Elastic-Net Regularization Linear Model (GIMNET), which is a package that fits the Generalized Linear model by punishing maximum likelihood (Friedman et al., 2010). The regularization path of the Lasso or elastic net penalty is calculated on the value grid of the regularization parameter. The algorithm is high-speed, can make full use of the sparsity of input matrix $\mathrm{X}$, and is suitable for linear, logic, polynomial, poisson and Cox regression models. It can also be applied to multi-response linear regression. These algorithms can process vast datasets and can make use of sparsity in the feature set.

$$
\min _{\beta_{0}, \beta} \frac{1}{N} \sum_{i=1}^{N} \omega_{i} l\left(y_{i}, \beta_{0}+\beta^{T} x_{i}\right)+\lambda\left[(1-\alpha)\|\beta\|_{2}^{2} / 2+\alpha\|\beta\|_{1}\right]
$$

Wherein the values of the grid $\lambda$ cover the entire range, $l\left(y_{i}, \eta_{i}\right)$ is the negative logarithmic likelihood distribution of the contribution to the observed value $i$. For example, it is $\frac{1}{2}(y-\eta)^{2}$ for Gaussian. The elastic mesh penalty is controlled by $\alpha$. (The specific formula refer to Friedman et al. (2010)). 


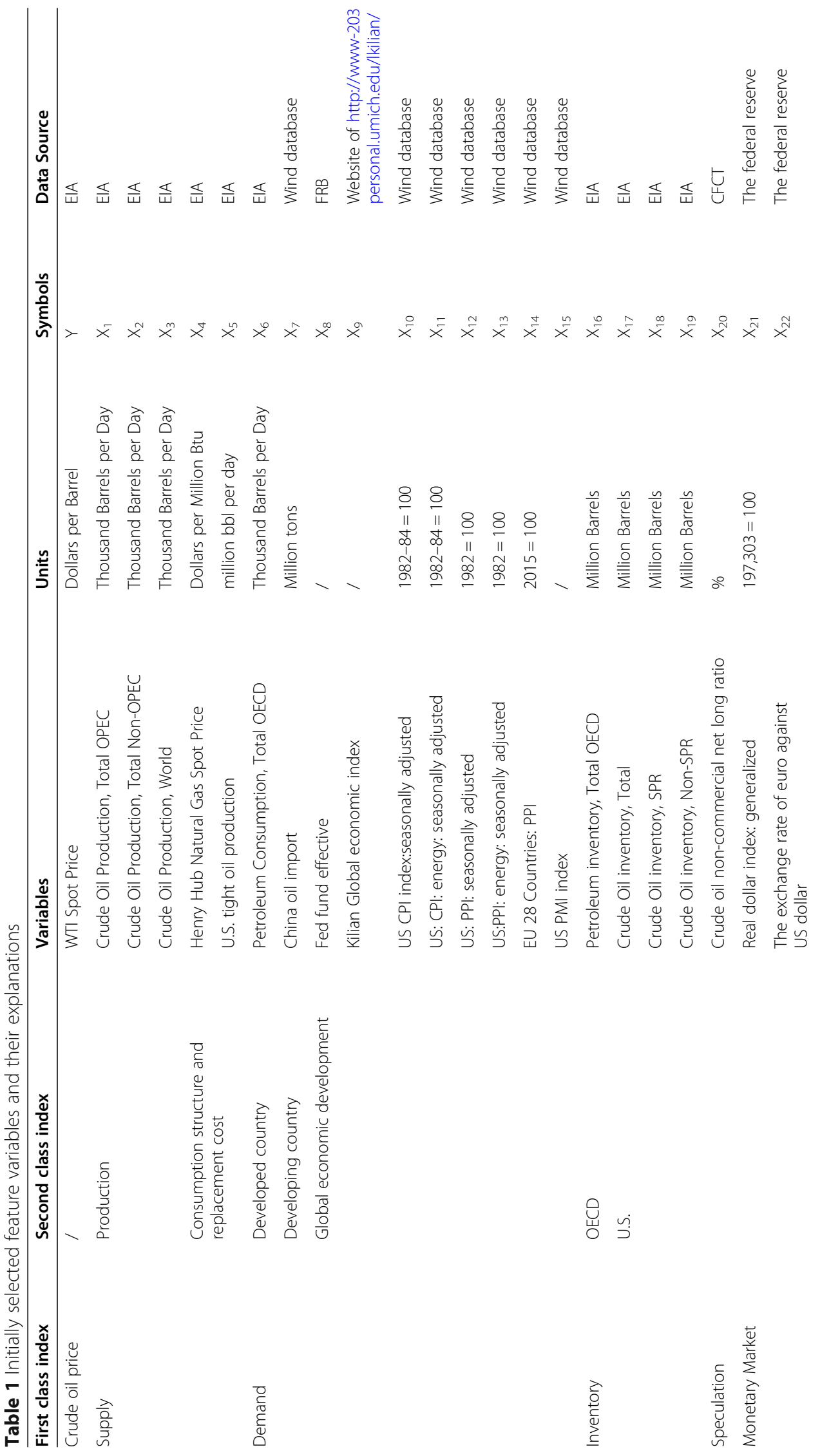




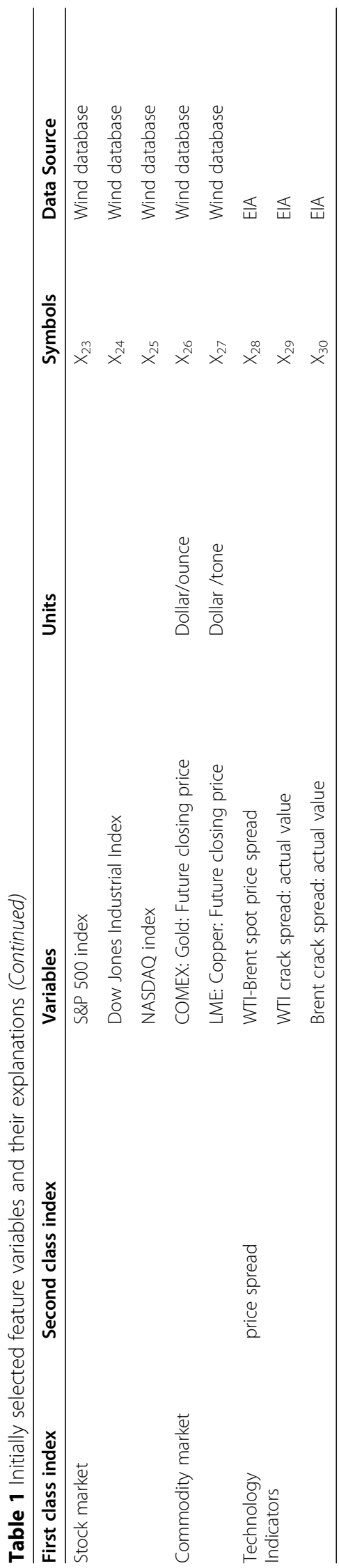




\section{Bayesian model averaging}

The basic idea of the Bayesian model averaging approach (BMA) can comprehend. Each model is not fully accepted or not entirely negative (Leamer, 1978). The prior probability of each model should be assumed firstly. The posterior probability can be gain by extracting the dataset contains information as well as the reception of models to the dependent variables. The excellence of the BMA approach is not only that it can sort the influence factors according to their importance but also can calculate the posterior mean, standard deviation and other indicators of the corresponding coefficients. With the help of the Markov chain Monte Carlo method (MCMC), the weight distribution of the model according to the prior information could be estimated (Godsill et al., 2001; Green, 1995). The MCMC method can overcome the shortcoming of BIC, AIC and EM methods. (The specific formula refer to (Leamer, 1978; Merlise, 1999; Raftery et al., 1997)).

It has the following three advantages: First, under different conditional probability distributions, there is no need to change the algorithm. Second, the posterior distribution of the weight and variance of BMA is considered comprehensively. Third, it can handle parameters with high BMA correlation.

\section{Spike-slab lasso}

Although model averaging can be considered a method of handling the variable selection and hypothesis testing task, only in a Bayesian context since model probabilities are required. Recently, Ročková, V. and George, E. I. introduce a new class of selfadaptive penalty functions that moving beyond the separable penalty framework based on a fully Bayes spike-and-slab formulation (Ročková \& George, 2018). Spike-slab Lasso (SSL) can borrow strength across coordinates, adapt to ensemble sparsity information and exert multiplicity adjustment by non-separable Bayes penalties. Meanwhile, it is using a sequence of Laplace mixtures with an increasing spike penalty $\lambda_{0}$, and keeping $\lambda_{1}$ fixed to a small constant. It is different from Lasso, which a sequence of single Laplace priors with an increasing penalty $\lambda$. Furthermore, it revisits deployed the EMVS procedure (an efficient EM algorithm for Bayesian model exploration with a Gaussian spike-and-slab mixture (Ročková \& George, 2018)) for SSL priors, automatic variable selection through thresholding, diminished bias in the estimation, and provably faster convergence. (The specific formula refer to (Ročková \& George, 2018)).

\section{Long short-term memory network}

Long short-term memory (LSTM) neural networks are a special kind of recurrent neural network (RNN). LSTM was initially introduced by Hochreiter and Schmidhuber (1997) (Hochreiter \& Schmidhuber, 1997), and the primary objectives of LSTM were to model long-term dependencies and determine the optimal time lag for time series issues. In this subsection, the architecture of RNN and its LSTM for forecasting crude oil prices are introduced. We start with the primary recurrent neural network and then proceed to the LSTM neural network.

The RNN is a type of deep neural network architecture with a deep structure in the temporal dimension. It has been widely used in time series modeling. A traditional neural network assumes that all units of the input vectors are independent of each other. As a result, the conventional neural network cannot make use of sequential 
information. In contrast, the RNN model adds a hidden state generated by the sequential information of a time series, with the output dependent on the hidden state. Figure 2 shows an RNN model being unfolded into a full network. The mathematical symbols in Fig. 2 are as follows:

1. $x_{t}$ denotes the input vector at time $t$.

2. $s_{t}$ denotes the hidden state at time $t$, which is determined relayed on the input vector $x_{t}$ and the previous hidden state. Then the hidden state $s_{t}$ is determined as follows:

$$
s_{t}=f\left(U x_{t}+W s_{t-1}\right)
$$

Where $f(\cdot)$ is the activation function, it has many alternatives such as sigmoid function and ReLU. The initial hidden state $s_{0}$ is usually initialized to zero.

3. $o_{t}$ denotes the output vector at time $t$. It can be calculated by:

$$
o_{t}=f\left(V s_{t}\right)
$$

4. $U$ and $V$ denote the weights of the hidden layer and the output layer respectively. $W$ denotes transition weights of the hidden state.

Although RNNs simulate time series data well, these are difficult to learn long-term dependence because of the vanishing gradient issue. LSTM is an effective way to solve the vanishing gradient by using memory cells. A memory cell is consists of four units: input gate, forget gate, output gate and a self-recurrent neuron, it is shown in Fig. 3. The gate controls the interactions between the adjacent memory cells and the memory cell itself. Whether the input signal can change the state of the memory cell is controlled by the input gate. On the other hand, the output gate can control the state of the memory cell to decide whether it can change the state of other memory cells. Additionally, the forget gate can choose to remember or forget its previous state.

Figure 4 illustrates the unrolled module in an LSTM network, which describes how the values of each gate are updated. The mathematical symbols in Fig. 4 are as follows:

1. $x_{t}$ is the input vector of the memory cell at time $t$.

2. $W_{i}, W_{f}, W_{c}, W_{o}, U_{i}, U_{f}, U_{c}, U_{o}$ and $V_{o}$ are weight matrices.

3. $b_{i}, b_{f}, b_{c}$ and $b_{o}$ are bias vectors.

4. $h_{t}$ is the value of the memory cell at time $t$.

5. $i_{t}$ and $\tilde{C}_{t}$ are values of the input gate and the candidate state of the memory cell at time $t$, respectively. $i_{t}$ and $\tilde{C}_{t}$ can be calculated by:

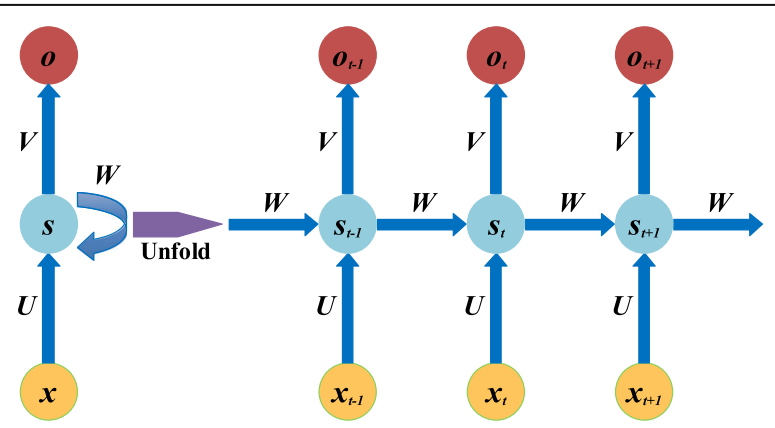

Fig. 2 An unrolled recurrent neural network 


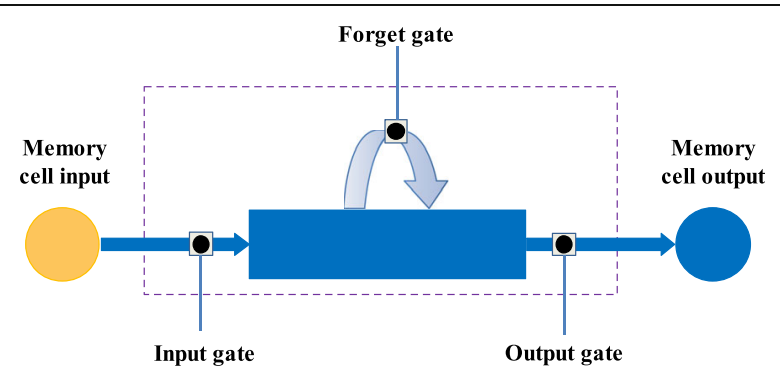

Fig. 3 The architecture of an LSTM memory cell

$$
\begin{aligned}
& i_{t}=\sigma\left(W_{i} x_{t}+U_{i} h_{t-1}+b_{i}\right) \\
& \tilde{C}_{t}=\tanh \left(W_{c} x_{t}+U_{c} h_{t-1}+b_{c}\right)
\end{aligned}
$$

6. $f_{t}$ and $C_{t}$ are values of the forget gate and the state of the memory cell at time $t$, respectively. $f_{t}$ and $C_{t}$ can be formulated as follows:

$$
\begin{aligned}
& f_{t}=\sigma\left(W_{f} x_{t}+U_{f} h_{t-1}+b_{f}\right) \\
& C_{t}=i_{t} * \tilde{C}_{t}+f_{t} * C_{t-1}
\end{aligned}
$$

7. $o_{t}$ and $h_{t}$ are values of the output gate and the value of the memory cell at time $t$, respectively. $o_{t}$ and $h_{t}$ can be calculated as follows:

$$
\begin{aligned}
& o_{t}=\sigma\left(W_{o} x_{t}+U_{o} h_{t-1}+V_{o} C_{t}+b_{o}\right) \\
& h_{t}=o_{h} * \tanh \left(C_{t}\right)
\end{aligned}
$$

The architecture of the LSTM neural network includes the number of hidden layers and the number of delays, which is the number of past data that account for training and testing. Currently, there is no rule of thumb to select the number of delays and hidden layers. In this work, the number of hidden layers and delays are set to 5 and 4 by trial and error. The back-propagation algorithm is employed to train the LSTM network and MLP neural network. The learning rate, batch size and number of epochs are 0.05, 60 and 1000, respectively. The speed of convergence is controlled by the learning rate, which is a decreasing function of time. Setting the number of epochs and the learning rate to 1000 and 0.05 can achieve the convergence of the training. The empirical result will become stable once convergence is achieved through the combinations of parameters are varied. Interesting readers may refer to (Hochreiter \& Schmidhuber, 1997) for more information.

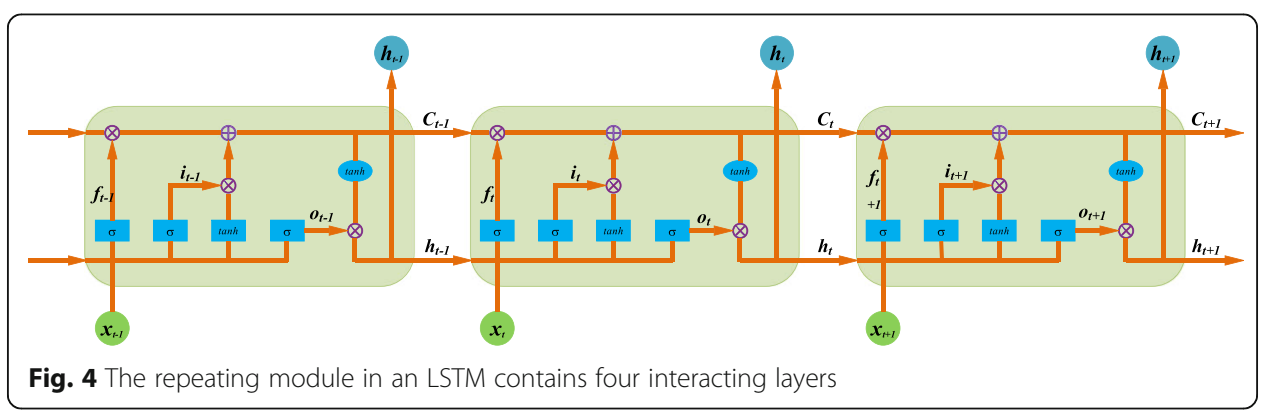




\section{Empirical study}

In this section, we compare the variable selection-LSTM integrated learning approach to the predictive performance of some benchmark models. First, section 4.1 analyzes the core influencing factors for the screening of the three feature extraction methods, and 4.2 describes the evaluation criteria and statistical tests to compare prediction accuracy. Second, section 4.3 provides an input selection and reference model parameter settings. Finally, Section 4.4 for the discussion.

\section{Variable selection}

We can see from Table 2, the elastic-net selects the most number (18 variables), followed by the SSL method (11 variables), and the BMA method includes the least number (8 variables). Meanwhile, the variable of the SSL method is a subset of the BMA method, and the BMA method is a subset of the elastic-net. Non-OPEC production, shale oil (tight oil) production, Fed Fund effective, Kilian Index, USA CPI, USA PPI: Energy, Euro PPI, USA: PMI, OECD inventory, USA SPR inventory, USA NonSPR inventory, Crude oil non-commercial net long ratio, Real dollar index: generalized, COMEX: Gold: Future closing price, LME: Copper: Future closing price, WTI-Brent spot price spread, WTI crack spread: actual value and Brent crack spread: real value. Firstly, we can see Non- OPEC production and tight oil production were selected from the supply aspect. It suggested that with the reduction of OPEC production, the production capacity of Non-OPEC countries is increasing gradually. In particular, the US tight oil production has become an essential factor affecting the trend of oil prices in recent years. According to the IEA report, the global oil production is expected to increase by 6.4 million barrels to 107 million barrels by 2023. The US tight oil production growth will account for 60 of the global growth. Meanwhile, in 2017, the US was the world's largest producer.

From the demand aspect, global economic development is still the main driver of crude oil price. The USA PPI: energy, Euro PPI and USA: PMI factors are selected by all three methods. Crude oil is an important raw material for industry, agriculture, and transportation. It is also the main parent product of energy and chemical products in the middle and lower reaches. Therefore, crude oil plays a critical role in the price of domestic production and living materials. For example, when the oil price continued to fall sharply, which is bound to lower the overall price level of the country. Moreover, it will have a heavier negative effect on domestic currency fluctuations, which will shift the currency situation from expansion to contraction.

From the inventory aspect, the OECD inventory, the USA SPR inventory and the USA Non-SPR inventory are chosen into the elastic-net model. The inventor is an indicator of the balance of the supply and demand for crude oil. Furthermore, the impact of commercial inventory on oil prices is much more substantial. When the future price

Table 2 Selected key features by GLMNET, SSL and BMA method

\begin{tabular}{ll}
\hline Methods & Selected feature ID \\
\hline GLMNET & $X_{2,}, X_{5}, X_{8}, X_{91}, X_{10}, X_{13}, X_{14}, X_{15}, X_{16}, X_{18}, X_{19}, X_{20}, X_{21}, X_{26}, X_{27}, X_{28}, X_{29}, X_{30}$ \\
Spike-slab Lasso & $X_{13}, X_{14}, X_{15}, X_{20}, X_{21}, X_{27}, X_{29}, X_{30}$ \\
Bayesian model averaging & $X_{13}, X_{14}, X_{15}, X_{16}, X_{18}, X_{19}, X_{20}, X_{21}, X_{27}, X_{29}, X_{30}$ \\
\hline
\end{tabular}


is much higher than the spot price, the oil companies tend to increase the commercial inventory, which stimulates the spot price to rise and reduces the price difference. When the future price is lower than spot prices, oil companies tend to reduce their commercial inventories, and spot prices fall, which will form a reasonable spread with futures prices.

From the speculate aspect, there is a positive correlation between the noncommercial net long ratio and oil price (Sanders et al., 2004). With the crude oil market and the stock market relation gradually strengthened, hedging plays a more important role in driving market trading (Coleman, 2012).

From the exchange rate market, the real dollar index: generalized was selected. The dollar index is used to measure the degree of change in the dollar exchange rate against a basket of currencies. If the dollar keeps falling, real revenues from oil products priced in dollars will fall, which will lead to the crude price high.

Form the commodity market, the LME: Copper: Future closing price factor is picked up. Copper has the function of resisting inflation, while the international crude oil price is closely related to the inflation level. There is an interaction between them from the long-term trend.

From the technical aspect, the WTI-Brent spread serves as an indicator of the tightening in the crude oil market. As the spread widens, which suggests the global supply and demand may be reached a tight equilibrium. The trend of price spread showed a significant difference around 2015 because of the US shale oil revolution. US shale production has surged substantially since 2014. However, due to the oil embargo, the excess US crude oil could not be exported, resulting in a significant increase in US Cushing crude oil inventories. The spread at this stage was mainly affected by the WTI price, and the correlation between the price difference and the oil price trend was not strong. After the United States lifted the ban on oil exports at the end of 2015, the correlation between the price spread and the oil price trend increased significantly. After the lifting of the oil export ban, the WTI oil price was no longer solely affected by the internal supply and demand of the United States. During this period, Brent and WTI oil prices were more consistent, resulting in a narrower spread. In addition, after 2015, the consistency of them increased significantly.

In summary, the factors selected by all three models are USA PPI: Energy, Euro PPI, USA: PMI (global economic development), Crude oil non-commercial net long ratio (speculate factor), Real dollar index: generalized (exchange rate market), LME: Copper: Future closing price (commodity market), WTI crack spread: actual value and Brent crack spread: actual value (technology factor).

\section{Evaluation criteria and statistic test}

To compare the forecasting performance of our proposed approach with some other benchmark models from level forecasting and directional forecasting, three main evaluation criteria, i.e., root mean squared error (RMSE), mean absolute percentage error (MAPE), directional symmetry (DS), which have been frequently used in recent years (Chiroma et al., 2015; Mostafa \& El-Masry, 2016; Yu et al., 2008b; Wang et al., 2017), are selected to evaluate the in-sample and out-of-sample forecasting performance. Three indicators are defined as follows: 


$$
\begin{aligned}
& \text { RMSE }=\sqrt{\frac{1}{N} \sum_{t=1}^{N}\left(x_{t}-\hat{x}_{t}\right)^{2}} \\
& M A P E=\frac{1}{N} \sum_{t=1}^{N}\left|\frac{x_{t}-\hat{x}_{t}}{x_{t}}\right| \times 100 \% \\
& D S=\frac{1}{T} \sum_{i=1}^{T} d_{i} \times 100 \%, \text { where } d_{i}= \begin{cases}1 & \text { if }\left(x_{i}-x_{i-1}\right)\left(\hat{x}_{i}-x_{i-1}\right) \geq 0 \\
0 & \text { otherwise }\end{cases}
\end{aligned}
$$

where $x_{t}$ and $\hat{x}_{t}$ denote the actual value and forecasted value at time $t$, respectively, $d_{i}=1$ if $\left(x_{i}-x_{i-1}\right)\left(\hat{x}_{i}-x_{i-1}\right) \geq 0$ or $d_{i}=0$ otherwise, and $N$ is total number of observations.

To provide statistical evidence of the forecasting performance of our proposed ensemble learning approach, three tests, i.e., the Diebold-Mariano (DM) (Diebold \& Mariano, 1995) test, the Superior Predictive Ability (Hansen, 2005) test and PesaranTimmermann (PT) (Pesaran \& Timmermann, 1992) test, are performed. The DM test checks the null hypothesis of equal predictive accuracy. In this study, the Mean Squared Error is applied as DM loss function and each model is compared against a random walk. As for performance measurement, we use the RMSE and MAPE. The PT test examines whether the directional movements of the real and forecast values are the same. In other words, it checks how well rises and falls in the forecasted value follow the actual rises and falls of the time series. The null hypothesis is that the model under study has no power in forecasting the oil prices.

\section{Forecasting performance evaluation}

To verify the predictive power of the variable Select-machine learning integrated approach, we selected 8 benchmark models, including 6 multivariate models (MLP, RBFNN, GRNN, ELMAN, WNN, ELM) and 2 univariate models (RW and ARMA). In this paper, the number of MLP output layer neurons is 1 , the number of iterations in the training stage is 10,000 , and the number of hidden layer neurons is determined by the trial and error method to be 11. Similarly, the number of LSTM hidden layers and the number of delays were set as 5 and 4, respectively, and the number of output layer neurons was set as 1 . The structure of the LSTM neural network was trained by backpropagation algorithm (BP), and the learning rate, batch size and the number of the epoch were set as 0.05, 60 and 1000 respectively. The convergence rate is controlled by the learning rate, which is a function of decreasing time. When the number and learning rate of epochs is set at 1000 and 0.05 , the convergence of the training set is realized, and the empirical results tend to be stable, which can recognize the convergence of the training set data. When the parameter combination changes, once it converges, the experimental results tend to be stable. All models are implemented using Matlab 2017B software.

According to the forecast results of Table 3, we can find some interesting conclusions: (1) no matter in the sample inside or outside the sample prediction, this chapter proposed variable selection - machine learning approach to integration in the training set and test set level precision (RMSE and MAPE) and direction (DS) were better than that of the single variable precision model and the core factors extracted model. (2) 
Table 3 Forecasting performance

\begin{tabular}{|c|c|c|c|c|c|c|c|}
\hline \multirow[t]{2}{*}{ Types } & \multirow[t]{2}{*}{ Models } & \multicolumn{3}{|c|}{ In-sample } & \multicolumn{3}{|c|}{ Out-of-sample } \\
\hline & & RMSE & MAPE (\%) & DS (\%) & RMSE & MAPE (\%) & DS (\%) \\
\hline \multirow[t]{2}{*}{ Time series } & RW & 4.89 & 4.02 & 49.42 & 5.74 & 5.19 & 48.84 \\
\hline & ARMA & 4.01 & 3.54 & 55.81 & 4.87 & 4.53 & 53.49 \\
\hline \multirow[t]{7}{*}{ Without variables selection } & MLP & 3.49 & 2.68 & 65.70 & 4.62 & 3.07 & 62.79 \\
\hline & RBFNN & 3.56 & 2.33 & 66.28 & 4.51 & 2.91 & 60.47 \\
\hline & GRNN & 3.36 & 2.37 & 66.86 & 4.48 & 2.85 & 62.79 \\
\hline & Elman & 2.93 & 1.79 & 69.19 & 3.61 & 2.21 & 67.44 \\
\hline & WNN & 2.39 & 1.61 & 70.35 & 3.44 & 1.78 & 67.44 \\
\hline & ELM & 2.26 & 1.52 & 71.51 & 3.11 & 1.63 & 69.77 \\
\hline & LSTM & 2.21 & 1.35 & 72.67 & 3.17 & 1.56 & 69.77 \\
\hline \multirow[t]{7}{*}{ GLMNET } & MLP & 3.58 & 2.17 & 66.86 & 4.49 & 2.82 & 60.47 \\
\hline & RBFNN & 3.36 & 2.18 & 69.17 & 4.29 & 2.72 & 65.12 \\
\hline & GRNN & 3.13 & 2.01 & 68.60 & 4.23 & 2.56 & 67.44 \\
\hline & Elman & 2.05 & 1.38 & 74.42 & 3.18 & 1.99 & 69.77 \\
\hline & WNN & 1.95 & 1.29 & 75.58 & 2.98 & 1.82 & 72.09 \\
\hline & ELM & 1.83 & 1.26 & 77.33 & 2.93 & 1.48 & 69.77 \\
\hline & LSTM & 1.88 & 1.17 & 78.49 & 2.76 & 1.43 & 74.42 \\
\hline \multirow[t]{7}{*}{ Spike-slab Lasso } & MLP & 3.02 & 2.11 & 66.28 & 4.12 & 2.74 & 65.12 \\
\hline & RBFNN & 2.59 & 1.72 & 69.77 & 3.57 & 2.43 & 67.44 \\
\hline & GRNN & 2.57 & 1.65 & 70.35 & 3.42 & 2.23 & 65.12 \\
\hline & Elman & 1.74 & 0.92 & 76.16 & 2.89 & 1.41 & 72.09 \\
\hline & WNN & 1.63 & 1.02 & 78.49 & 2.91 & 1.45 & 74.42 \\
\hline & ELM & 1.57 & 0.95 & 80.23 & 2.62 & 1.31 & 72.09 \\
\hline & LSTM & 1.48 & 0.85 & 80.81 & 2.45 & 1.02 & 76.74 \\
\hline \multirow[t]{7}{*}{ Bayesian model averaging } & MLP & 2.81 & 1.97 & 70.93 & 3.92 & 2.64 & 67.44 \\
\hline & RBFNN & 2.25 & 1.66 & 75.00 & 3.41 & 2.23 & 72.09 \\
\hline & GRNN & 2.05 & 1.52 & 74.42 & 3.18 & 2.09 & 69.77 \\
\hline & Elman & 1.28 & 0.95 & 78.49 & 2.43 & 1.01 & 76.74 \\
\hline & WNN & 1.21 & 0.92 & 82.56 & 2.08 & 1.05 & 74.42 \\
\hline & ELM & 1.29 & 0.78 & 83.72 & 2.13 & 0.91 & 76.74 \\
\hline & LSTM & 1.12 & 0.74 & 84.88 & 2.04 & 0.83 & 81.40 \\
\hline
\end{tabular}

Note: The table reports the RMSE values of each model forecast, while the PT statistics are in the parenthesis. ${ }^{* *}$ denotes a rejection of the null hypothesis at the $1 \%$ significance level

Among the variable selection-machine learning integration models, the BMA-LSTM integration model performs best, followed by Spike and Slab LASSO-LSTM and GLMN ET-LSTM. For example, the RMSE, MAPE and DS predicted in the SAMPLE of rhe BMA-LSTM integrated module were $1.12 \%, 0.74 \%$ and $84.88 \%$, respectively, which were $3.77 \%, 3.28 \%$ and $35.46 \%$ higher than RW, and $1.09 \%, 0.61 \%$ and $12.21 \%$ higher than the LSTM model without variable selection. (3) When predicted one step in advance, RMSE, MAPE and DS of the BMA-LSTM integrated model were 2.04\%, 0.83\% and $81.40 \%$, respectively, $3.7 \%, 4.36 \%$ and $32.56 \%$ higher than that of the RW model, and $1.13 \%, 0.73 \%$ and $11.63 \%$ higher than that of the variable free LSTM model. It shows that the prediction accuracy of the variable selection-machine learning integrated model is significantly improved compared with that of the univariate model and 
the univariate model. Secondly, the number of core variables selected by BMA is neither the most nor the least among the three variable selection models, indicating that the number of core variables will also affect the prediction results.

\section{Statistic tests}

According to Table 4 statistical test results can be seen that (1) the step ahead prediction samples, variable selection - machine learning integration model of DM test results are less than 7.195, this means that the performance of the proposed method under the confidence level of $99 \%$ is better than all the other benchmark model, the possible reason is the variable selection - machine learning integration model significantly improves

Table 4 The results of Diebold-Mariano and PT statistics

\begin{tabular}{|c|c|c|c|c|c|}
\hline \multirow[t]{2}{*}{ Types } & \multirow[t]{2}{*}{ Models } & \multicolumn{2}{|l|}{ In-sample } & \multicolumn{2}{|c|}{ Out-of-sample } \\
\hline & & DM & PT & DM & PT \\
\hline \multirow[t]{2}{*}{ Time series } & RW & - & 1.025 & - & 0.984 \\
\hline & ARMA & $-2.053 *$ & $2.178 *$ & -1.784 * & 1.946 \\
\hline \multirow[t]{7}{*}{ Without variables selection } & MLP & $-7.107 * *$ & $3.372 * *$ & $-6.543 * *$ & $2.521 *$ \\
\hline & RBFNN & $-7.314 * *$ & $3.451 * *$ & $-6.745^{* *}$ & $2.873 * *$ \\
\hline & GRNN & $-7.943 * *$ & $3.233 * *$ & $-7.153 * *$ & $3.084 * *$ \\
\hline & Elman & $-8.017 * *$ & $5.484^{* *}$ & $-7.491 * *$ & $4.141 * *$ \\
\hline & WNN & $-7.462 * *$ & $4.177^{* *}$ & $-6.897 * *$ & $3.052 * *$ \\
\hline & ELM & $-7.516 * *$ & $4.635 * *$ & $-6.983 * *$ & $3.314 * *$ \\
\hline & LSTM & $-8.214^{* *}$ & $4.464^{* *}$ & $-7.635^{* *}$ & $3.633 * *$ \\
\hline \multirow[t]{7}{*}{ GLNET } & MLP & $-8.425 * *$ & $3.951 * *$ & $-7.849 * *$ & $4.687 * *$ \\
\hline & RBFNN & $-7.566 * *$ & $4.130^{* *}$ & $-6.951 * *$ & $3.892 * *$ \\
\hline & GRNN & $-7.847^{* *}$ & $4.478 * *$ & $-7.283 * *$ & $4.174 * *$ \\
\hline & Elman & $-8.362 * *$ & $5.632 * *$ & $-7.719 * *$ & $3.952 * *$ \\
\hline & WNN & $-8.891 * *$ & $4.655 * *$ & $-8.206 * *$ & $5.613 * *$ \\
\hline & ELM & $-7.195 * *$ & $4.553 * *$ & $-6.618 * *$ & $2.370 * *$ \\
\hline & LSTM & $-7.219 * *$ & $4.468 * *$ & $-6.806 * *$ & $2.855 * *$ \\
\hline \multirow[t]{7}{*}{ Spike-slab Lasso } & MLP & $-7.873^{* *}$ & $4.021 * *$ & $-7.194^{* *}$ & $3.324 * *$ \\
\hline & RBFNN & $-8.129 * *$ & $5.566^{* *}$ & $-7.506 * *$ & $4.068 * *$ \\
\hline & GRNN & $-7.502^{* *}$ & $5.171 * *$ & $-6.904 * *$ & $3.281 * *$ \\
\hline & Elman & $-7.604^{* *}$ & $5.044 * *$ & $-6.816^{* *}$ & $3.693 * *$ \\
\hline & WNN & $-8.138 * *$ & $4.936 * *$ & $-7.728 * *$ & $3.586 * *$ \\
\hline & ELM & $-8.608 * *$ & $6.052 * *$ & $-7.893 * *$ & $5.024 * *$ \\
\hline & LSTM & $-7.672 * *$ & $6.843 * *$ & $-7.015^{* *}$ & $4.338 * *$ \\
\hline \multirow[t]{7}{*}{ Bayesian model averaging } & MLP & $-7.748 * *$ & $5.167 * *$ & $-7.296 * *$ & $4.844 * *$ \\
\hline & RBFNN & $-8.424 * *$ & $5.023 * *$ & $-7.693 * *$ & $4.463 * *$ \\
\hline & GRNN & $-8.795 * *$ & $6.431 * *$ & $-8.334^{* *}$ & $5.572 * *$ \\
\hline & Elman & $-7.796 * *$ & $6.082 * *$ & $-7.258 * *$ & $5.185 * *$ \\
\hline & WNN & $-8.013 * *$ & $7.690 * *$ & $-7.374^{* *}$ & $5.641 * *$ \\
\hline & ELM & $-8.507 * *$ & $7.724^{* *}$ & $-7.867 * *$ & $6.030 * *$ \\
\hline & LSTM & $-9.304 * *$ & $8.035 * *$ & $-8.746 * *$ & $6.544 * *$ \\
\hline
\end{tabular}

Note: The values in the table are the calculated DM statistics. * denotes a rejection of null hypothesis at the $5 \%$ significance level. ** denotes a rejection of null hypothesis at the $1 \%$ significance level 
the prediction performance of the model. (2) In the out-of-sample prediction 1 step in advance, when the LR model is used as the test model, the DM test results of other test models are far less than -7.015 , indicating that the predictive performance of the three integrated methods and the other three single models is better than that of the machine learning model without variable selection at the $99 \%$ confidence level. (3) samples within 1 step ahead prediction and sample 1 step ahead prediction of the performance of the three variables extraction method was compared, the BMA-LSTM integration model to predict performance is the best, the next step is the Spike and slab LASSOLSTM and GLMNET-LSTM, suggesting that this chapter puts forward the integrated research framework based on the variable selection-machine learning significantly improves the performance of the integrated machine learning approach.

PT test results also give three interesting points: (1) In the one step forward insample forecasting, the PT test results of the proposed variable selection-machine learning integration approach are all rejected the movement direction of the actual independence assumption under $99 \%$ confidence level. This also means that the variable selection-machine learning method is the best direction prediction performance and also can be seen that the direction of the ARIMA predicts performance is the worst. (2) In the out-of-sample one-step forecasting, the PT test value of the predicted results of the integrated method is significantly greater than that of the single model, which means that the direction prediction ability of the integrated method is better than that of the single model, mainly because the variable selection-machine learning integration idea significantly improves the direction prediction performance of the single model. (3) In the prediction in and out of sample 1 step in advance, it can be seen from the PT test results of variable Selection-machine learning integration method that the direction prediction accuracy of BMA-LSTM is the highest, followed by Spike-Slab LassoLSTM, which is mainly attributed to the direction prediction ability of variable Selection-machine learning method.

\section{Conclusions and future work}

In this paper, we proposed a variable selection and machine learning framework that combines the variable selection (BMA) and forecasting method (LSTM) to forecast the oil price and compared its forecasting performance with other primary and new variable selection methods (elastic-net and spike and slab Lasso). Moreover, compared to other popular benchmark forecast methods (RW, ARMA, MLP, RBFNN, GRNN, EIMAN, WNN, ELM). Specifically, our contributions are as follow:

Introduce the variable selection before forecasting. In this process, we compare three different methods and analyze core influencing factors based on the literature review from supply and demand, global economic development, financial market, and technology aspects. The results showed that the variable of the SSL method is a subset of the BMA method, and the BMA method is a subset of the elastic-net.

Testing the performance of the proposed variable selection and machine learning framework based on 3 variable selections and 8 individual forecasts. Comparing with the 8 individual forecasts without variable selection, the combinations forecasting reduces the errors. The results showed that (1) the variable choice-machine learning integration method proposed in this chapter is superior to the univariate model and the model without core factor extraction in both training set and test set level accuracy 
(RMSE, MAPE) and direction symmetric (DS). (2) Among the variable selectionmachine learning integration models, The BMA-LSTM integration model performs best, followed by Spike and Slab LASSO-LSTM and GLMNET-LSTM. It shows that the prediction accuracy of the variable selection-machine learning integrated model is significantly improved compared with that of the univariate model and the univariate model. Secondly, the number of core variables selected by BMA is neither the most nor the least among the three variable selection models, indicating that the number of core variables will also affect the prediction results. (3) The statistical test results show that the prediction of 1 step in advance in-sample and 1 step in advance in out of sample. Compared with the prediction performance of the three variable extraction methods, the directional prediction accuracy and horizontal prediction accuracy of the BMALSTM integrated model are the best, followed by Spike and Slab-LASSO-LSTM and GLMNET-LSTM. This indicates that the variable selection-based machine learning integrated research framework proposed in this chapter significantly improves the forecasting performance of oil prices. In future research, we may introduce more independent variables with the help of internet search data, test our framework performance. Moreover, investor sentiment can be quantified in this process. In addition, different variable selection methods can be introduced more.

\begin{abstract}
Abbreviations
ANNs: artificial neural networks; ARMA: autoregressive moving average; ARIMA: autoregressive integrated moving average; BMA: Bayesian model averaging; DM: Diebold-Mariano; DS: directional symmetry; EMD: empirical mode decomposition; ENN: elman neural Networks; ETS: exponential smoothing; FNN: feed-forward neural network; GA: genetic algorithms; GARCH: generalized autoregressive conditional heteroskedastic model; GLMNET: elastic-net regularized generalized linear Model; GRNN: generalized regression neural network Models; LSTM: long short-term memory; MIMO: multiple-input multiple-output; MAPE: mean absolute percentage error; MCMC: Markov chain Monte Carlo method; PT: Pesaran-Timmermann; RMSE: root mean squared error; RNN: recurrent neural network; RW: random walk; RWM: random walk model; SBM: slope-based method; SDAE: stacked denoising autoencoders model; SSL: Spikeslab Lasso; SVM: support vector machines; VAR: vector autoregression model; WNN: Walvet Neural Networks; WTI: West Texas Intermediate
\end{abstract}

\title{
Supplementary Information
}

The online version contains supplementary material available at https://doi.org/10.1186/s42162-021-00166-4.

Additional file 1. Neural networks training characteristics. Table A.1. Neural networks design and training characteristics

About this supplement

This article has been published as part of Energy Informatics Volume 4, Supplement 2 2021: Proceedings of the Energy Informatics.Academy Conference Asia 2021. The full contents of the supplement are available at https:// energyinformatics.springeropen.com/articles/supplements/volume-4-supplement-2.

\section{Authors' contributions}

Q. L.: Data curation, Conceptualization, Methodology, Software, Writing - original draft, Funding acquisition; S. S.: Methodology, Writing - review\& editing; H. D.: Writing - review\& editing, Funding acquisition.; S. W.: Conceptualization, Supervision,Validation, Funding acquisition. All co-authors have read and approved the final manuscript.

\section{Funding}

This research is supported by the National Natural Science Foundation of China (71988101; 71874177 and

72022019), China Postdoctoral Science Foundation (2020 M68719), and the University of Chinese Academy of Sciences.

Availability of data and materials

Not applicable.

\section{Declarations}

Ethics approval and consent to participate

Not applicable. 


\section{Competing interests}

The authors declare that they have no competing interests.

\section{Author details}

${ }^{1}$ Institute of Systems Science, Academy of Mathematics and Systems Science, Chinese Academy of Sciences, Beijing 100190, China. ${ }^{2}$ The School of Management Xi'an Jiaotong University Xi'an 710049 China . ${ }^{3}$ School of Economics and Management, University of Chinese Academy of Sciences, Beijing 100190, China.

\section{Published: 24 September 2021}

\section{References}

Barsky RB, Kilian L (2001) Do we really know that oil caused the great stagflation? A monetary alternative. NBER Macroecon Annu 16:137-183. https://doi.org/10.1086/654439

Baumeister C, Kilian L, Zhou X (2013) Are product spreads useful for forecasting? An empirical evaluation of the verleger hypothesis. Available at SSRN DP9572

Castle JL, Qin X, Reed WR (2009) How to pick the best regression equation: a review and comparison of model selection algorithms. Working Papers in Economics 32(5):979-986

Chai J, Xing LM, Zhou XY, Zhang ZG, Li JX (2018) Forecasting the WTI crude oil price by a hybrid-refined method. Energy Econ 71:114-127. https://doi.org/10.1016/j.eneco.2018.02.004

Charles A, Darné O (2017) Forecasting crude-oil market volatility: further evidence with jumps. Energy Econ 67:508-519. https://doi.org/10.1016/j.eneco.2017.09.002

Chiroma H, Abdulkareem S, Herawan T (2015) Evolutionary neural network model for West Texas intermediate crude oil price prediction. Appl Energy 142:266-273. https://doi.org/10.1016/j.apenergy.2014.12.045

Cifarelli G, Paladino G (2010) Oil price dynamics and speculation: a multivariate financial approach. Energy Econ 32(2):363372. https://doi.org/10.1016/j.eneco.2009.08.014

Coleman L (2012) Explaining crude oil prices using fundamental measures. Energy Policy 40:318-324. https://doi.org/10.1016/ j.enpol.2011.10.012

Diebold FX, Mariano RS (1995) Comparing predictive accuracy. J Bus Econ Stat 20(1):134-144

Doroodian K, Boyd R (2003) The linkage between oil price shocks and economic growth with inflation in the presence of technological advances: a CGE model. Energy Policy 31(10):989-1006. https://doi.org/10.1016/S0301-4215(02)00141-6

Drachal K (2016) Forecasting spot oil price in a dynamic model averaging framework-have the determinants changed over time? Energy Econ 60:35-46. https://doi.org/10.1016/j.eneco.2016.09.020

Drezga I, Rahman S (1998) Input variable selection for ANN-based short-term load forecasting. IEEE Transactions on Power Systems Pwrs 13(4):1238-1244

Friedman J, Hastie T, Tibshirani R (2010) Regularization paths for generalized linear models via coordinate descent. J Stat Softw 33(1):1-22

Godsill S, Doucet A, West M (2001) Maximum a posteriori sequence estimation using Monte Carlo particle filters. Ann Inst Stat Math 53(1):82-96. https://doi.org/10.1023/A:1017968404964

Green PJ (1995) Reversible jump Markov chain Monte Carlo computation and Bayesian model determination. Biometrika 82(4):711-732. https://doi.org/10.1093/biomet/82.4.711

Hamilton JD (2009a) Understanding crude oil prices. Energy J 30(2):179-207

Hamilton JD (2009b) Causes and consequences of the oil shock of 2007-08 (no. w15002). National Bureau of economic research

Hansen PR (2005) A test for superior predictive ability. J Bus Econ Stat 23(4):365-380. https://doi.org/10.1198/0735001 05000000063

Hochreiter S, Schmidhuber J (1997) LSTM can solve hard long time lag problems. Adv Neural Inf Proces Syst:473-479

Huang T, Fildes R, Soopramanien D (2014) The value of competitive information in forecasting FMCG retail product sales and the variable selection problem. Eur J Oper Res 237(2):738-748. https://doi.org/10.1016/j.ejor.2014.02.022

Ji Q, Fan Y (2016) Evolution of the world crude oil market integration: a graph theory analysis. Energy Econ 53:90-100. https://doi.org/10.1016/j.eneco.2014.12.003

Kilian L (2009) Not all oil price shocks are alike: disentangling demand and supply shocks in the crude oil market. Am Econ Rev 99(3):1053-1069. https://doi.org/10.1257/aer.99.3.1053

Kilian L (2010) Explaining fluctuations in gasoline prices: a joint model of the global crude oil market and the US retail gasoline market. Energy $\mathrm{J}: 87-112$

Kilian L (2017) How the tight oil boom has changed oil and gasoline markets Social Science Electronic Publishing Available at SSRN No:6380

Kilian L, Hicks B (2013) Did unexpectedly strong economic growth cause the oil price shock of 2003-2008? J Forecast 32(5): 385-394. https://doi.org/10.1002/for.2243

Kilian L, Murphy DP (2014) The role of inventories and speculative trading in the global market for crude oil. J Appl Econ 29(3):454-478. https://doi.org/10.1002/jae.2322

Korobilis D (2013) VAR forecasting using Bayesian variable selection. J Appl Econ 28(2):204-230. https://doi.org/10.1 002/jae.1271

Leamer EE (1978) Specification searches. Wiley, New York

May RJ, Dandy GC, Maier HR, Nixon JB (2008) Application of partial mutual information variable selection to ANN forecasting of water quality in water distribution systems. Environ Model Softw 23(10-11):1289-1299. https://doi.org/10.1016/j. envsoft.2008.03.008

Merlise A (1999) Bayesian model averaging and model search strategies. Bayesian Statistics 6:157

Mostafa MM, El-Masry AA (2016) Oil price forecasting using gene expression programming and artificial neural networks. Econ Model 54:40-53. https://doi.org/10.1016/j.econmod.2015.12.014

Murat A, Tokat E (2009) Forecasting oil price movements with crack spread futures. Energy Econ 31(1):85-90. https://doi.org/1 0.1016/j.eneco.2008.07.008 
Narayan PK, Narayan S, Zheng X (2010) Gold and oil futures markets: are markets efficient? Appl Energy 87(10):3299-3303. https://doi.org/10.1016/j.apenergy.2010.03.020

Özbek L, Özlale Ü (2010) Analysis of real oil prices via trend-cycle decomposition. Energy Policy 38(7):3676-3683. https://doi. org/10.1016/j.enpol.2010.02.045

Pesaran MH, Timmermann A (1992) A simple nonparametric test of predictive performance. J Bus Econ Stat 10(4):461-465

Raftery AE, Madigan D, Hoeting JA (1997) Bayesian model averaging for linear regression models. J Am Stat Assoc 92(437): 179-191. https://doi.org/10.1080/01621459.1997.10473615

Reboredo JC (2012) Modelling oil price and exchange rate co-movements. J Policy Model 34(3):419-440. https://doi.org/10.1 016/j.jpolmod.2011.10.005

Ročková V, George El (2018) The spike-and-slab lasso. J Am Stat Assoc 113(521):431-444. https://doi.org/10.1080/01621459.2 016.1260469

Sadorsky P (1999) Oil price shocks and stock market activity. Energy Econ 21(5):449-469. https://doi.org/10.1016/50140-9883 (99)00020-1

Sanders DR, Boris K, Manfredo M (2004) Hedgers, funds, and small speculators in the energy futures markets: an analysis of the CFTC's commitments of traders reports. Energy Econ 26(3):425-445. https://doi.org/10.1016/j.eneco.2004.04.010

Sari R, Hammoudeh S, Soytas U (2010) Dynamics of oil price, precious metal prices, and exchange rate. Energy Econ 32(2): 351-362. https://doi.org/10.1016/i.eneco.2009.08.010

Suganthi L, Samuel AA (2012) Energy models for demand forecasting-a review. Renew Sust Energ Rev 16(2):1223-1240. https://doi.org/10.1016/.j.ser.2011.08.014

Sun S, Wei Y, Tsui KL, Wang S (2019) Forecasting tourist arrivals with machine learning and internet search index. Tour Manag 70:1-10. https://doi.org/10.1016/.jtourman.2018.07.010

Tang L, Yu L, Wang S, Li J, Wang S (2012) A novel hybrid ensemble learning paradigm for nuclear energy consumption forecasting. Appl Energy 93:432-443. https://doi.org/10.1016/j.apenergy.2011.12.030

Valgaev O, Kupzog F, Schmeck H (2020) Adequacy of neural networks for wide-scale day-ahead load forecasts on buildings and distribution systems using smart meter data. Energy Informatics 3(1):1-17

Wang J, Li X, Hong T, Wang S (2018) A semi-heterogeneous approach to combining crude oil price forecasts. Inf Sci 460:279-292

Wang Q, Sun X (2017) Crude oil price: demand, supply, economic activity, economic policy uncertainty and wars-from the perspective of structural equation modelling (SEM). Energy 133:483-490. https://doi.org/10.1016/j.energy.2017.05.147

Wang Y, Liu L, Wu C (2017) Forecasting the real prices of crude oil using forecast combinations over time-varying parameter models. Energy Econ 66:337-348. https://doi.org/10.1016/j.eneco.2017.07.007

Wang Y, Wu C, Yang L (2015) Forecasting the real prices of crude oil: a dynamic model averaging approach. Available at SSRN 2590195

Wang Y, Wu C, Yang L (2016) Forecasting crude oil market volatility: a Markov switching multifractal volatility approach. Int J Forecast 32(1):1-9. https://doi.org/10.1016/j.jiforecast.2015.02.006

Xiong T, Bao Y, Hu Z (2013) Beyond one-step-ahead forecasting: evaluation of alternative multi-step-ahead forecasting models for crude oil prices. Energy Econ 40(2):405-415. https://doi.org/10.1016/j.eneco.2013.07.028

Yu L, Wang S, Lai KK (2008a) Forecasting crude oil price with an EMD-based neural network ensemble learning paradigm. Energy Econ 30(5):2623-2635. https://doi.org/10.1016/j.eneco.2008.05.003

Yu L, Wang S, Lai KK (2008b) Forecasting crude oil price with an EMD-based neural network ensemble learning paradigm. Energy Econ 30(5):2623-2635. https://doi.org/10.1016/j.eneco.2008.05.003

Yu L, Wang Z, Tang L (2015) A decomposition-ensemble model with data-characteristic-driven reconstruction for crude oil price forecasting. Appl Energy 156:251-267. https://doi.org/10.1016/j.apenergy.2015.07.025

Yu L, Zhao Y, Tang L, Yang Z (2019) Online big data-driven oil consumption forecasting with Google trends. Int J Forecast 35(1):213-223. https://doi.org/10.1016/j.ijforecast.2017.11.005

Zhang JL, Zhang YJ, Zhang L (2015) A novel hybrid method for crude oil price forecasting. Energy Econ 49:649-659. https:// doi.org/10.1016/j.eneco.2015.02.018

Zhang X, Lai KK, Wang SY (2008) A new approach for crude oil price analysis based on empirical mode decomposition. Energy Econ 30(3):905-918. https://doi.org/10.1016/j.eneco.2007.02.012

Zhang Y, Ma F, Wang Y (2019) Forecasting crude oil prices with a large set of predictors: can LASSO select powerful predictors? J Empir Financ 54:97-117. https://doi.org/10.1016/j.jempfin.2019.08.007

Zhang YJ (2013) Speculative trading and WTI crude oil futures price movement: an empirical analysis. Appl Energy 107:394402. https://doi.org/10.1016/j.apenergy.2013.02.060

Zhang YJ, Chevallier J, Guesmi K (2017) "De-financialization" of commodities? Evidence from stock, crude oil and natural gas markets. Energy Econ 68:228-239

Zhang YJ, Wei YM (2011) The dynamic influence of advanced stock market risk on international crude oil returns: an empirical analysis. Quantitative Finance 11(7):967-978. https://doi.org/10.1080/14697688.2010.538712

Zhao Y, Li J, Yu L (2017) A deep learning ensemble approach for crude oil price forecasting. Energy Econ 66:9-16. https://doi. org/10.1016/.eneco.2017.05.023

Zhu B, Han D, Wang P, Wu Z, Zhang T, Wei YM (2017) Forecasting carbon price using empirical mode decomposition and evolutionary least squares support vector regression. Appl Energy 191:521-530. https://doi.org/10.1016/j.apenergy.2017. 01.076

Zou H, Hastie T (2005) Regularization and variable selection via the elastic net. Journal of the Royal Statistical Society: Series B (Statistical Methodology) 67(2):301-320. https://doi.org/10.1111/j.1467-9868.2005.00503.x

\section{Publisher's Note}

Springer Nature remains neutral with regard to jurisdictional claims in published maps and institutional affiliations. 